\title{
The Vehicle Routing Problem Complex Network Analysis
}

\author{
Gabriel Policroni ades Chípuli , Idalia Flores de la Mota, Olivia Sashiko Sihirai Reyna, Javier Lara de Paz
}

Universidad Nacional Autónoma de México.

How to cite this paper: Chípuli, G.P., de la Mota, I.F., Reyna, O.S.S., de Paz, J.L. (2018) The Vehicle Routing Problem Complex Network Analysis. Journal of Applied Mathematics and Computation, 2(12), 525-542.

DOI: $10.26855 /$ jamc.2018.12.001

*Corresponding author: Gabriel Po-

licroniades Chípuli, Universidad Nacional Autónoma de México.

Email: gpolicroniadesch@gmail.com

\section{Abstract}

One of the main characteristics of transportation problems is not only the hard design model process; but also, in the characterization of the elements that make up the supply chain. A more detailed analysis will grant undoubtedly a greater knowledge of the system. However, the use of regular networks for the development of models can eliminate key characteristics for correct decision making. Faced with this fact, the characterization of transport models is proposed through the conceptualization of complex networks. This new proposal will allow to apply metrics and perform more detailed analysis to the distribution network; such as vulnerability analysis, fault propagation, element grouping identification, among other metrics. The objective of this research is to show a methodology to build the Vehicle Routing Problem models and analyze the distribution network using the corresponding metrics of complex networks.

Keywords

VRP, Complex Networks, Logistic.

\section{Introduction.}

One of the great challenges of the industrial sector is undoubtedly the logistical aspect: the transfer of the product through the supply chain [24]. This key and decisive factor influences the final cost of the product however, among the activities carried out in the supply chain, it is considered as a necessary activity that does not generate more value to the product. This is the reason why, its cost seeks to be minimized $[17,26,40]$.

Transport models as mentioned in [21]; can be solved by the known VRP (Vehicle Routing Problem) which its objective is to find the optimal set of routes for a fleet of vehicles that must meet the demands of a given set of customers. That is a generalization of the well-known Traveling Salesman Problem (TSP, for its acronym in English).

An important characteristic [37] is that in most cases when VRP models are built, restrictions of the elements that make up the distribution network or characteristics of the environment are often omitted, which can lead to transport models with multiple objectives or with a high degree of computational complexity.

In [38] was written that a distribution network needs to be analyzed from a more complete perspective to give an overview of the network elements behavior for example, grouping of its elements, detection of bottlenecks, workloads balance between warehouses, as well as other metrics that can be applied before the development of a mathematical model. However, the same author mentions the necessity to increase the detail of traditional networks to obtain a more specific of the characteristics of each element that makes up the network. This new perspective is only achieved if the use of the complex network concept in the VRP model is generalized; as proposed in this article. 
The scope of the research consists in showing the proposed methodology and justifying its contribution. In a future research, examples will be made that show the advantages and disadvantages of its application.

This paper is organized as follows: the first section presents a brief introduction to the networks and their relationship with the problem of vehicle routing, addressing in a general way the problems to be solved by means of the proposed methodology. In section two the problem is addressed from the state of the art with a description about the Vehicle Routing Problem, the way in which they have been solved from the point of view of the traditional networks and the contribution of the theme, as a Novel factor that has not been explored and applied to VRP. In section three, the proposed methodology is described in a general way. Section four mentions the expected results and future work. Finally, section five presents the conclusions.

\section{State Of The Art}

The following state of the art is divided into two sections: the first shows a brief introduction to the problem of vehicle routing; and the second, addresses the concept of complex network and VRP.

\subsection{Brief description of the Vehicle Routing Problem}

One of the first approaches to the VRP problem was undoubtedly the one developed by [22], their model and algorithm were initially designed to solve a fuel distribution problem; This process was carried out by delivering gasoline to a group of customers located at different points. However, over time, a large number of restrictions have been added that have led to a wide variety of transportation models; as described in $[21,36,41,44,45,48]$.

It is important to mention that the VRP model is NP-hard [39,51] as these authors refer it among others, which means that they are classified as combinatorial problems with a non-polynomial computational complexity.

In [41] is defined the VRP as a complete network $G=(V, E)$, where $V=\{0,1, \ldots, n\}$ is the set of nodes, which represents the set of clients, node 0 represents the central store; assigned to these client nodes, there is a non-negative demand value $d_{j}$. The set $E$, is the set of $\operatorname{arcs}(i, j) \in E$; so that if there is an arc between $i$ and $j$, it means that there is a path between them. Assigned to each of these arcs, there is a cost $c_{i j}$ that will be equivalent to the transfer cost between node $i$ and node $j$. The objective of the VRP is to find a route at the minimum cost by visiting each of the customers with satisfaction of the total demand. This type of models has been solved by applying different optimization techniques. However, the most used for this type of model are the heuristic and metaheuristic methods [21].

A complete classification of the different VRP models can be found in [15] and [46]; however, new models and categories have emerged over time based on the need to model new real problems. In [41] is showed a VRP classification based on its topology. On the other hand, same authors mention that, depending on the restrictions of the problem and the characteristics of the system, it is possible to categorize this type of models.

The VRP models, as mentioned in [10,43], can be classified into two main branches, according to the characteristics and restrictions for which they were created. The first is identified by the authors as classic or deterministic models, which are based on the omission of unexpected factors that may put the reliability of the model at risk. The second one, stochastic models, is more attached to reality, where multiple factors are no longer controllable for both the modeler and the decision maker, as also mentioned by [32]. This is synthesized in four different categories as shown in Table 1

Table 1. Types of Stochastic Vehicle Routing Problems, source [32].

\begin{tabular}{|c|c|c|c|}
\hline Acronyms & Stochastic Parameter & Acronyms & Stochastic Parameter \\
\hline VRPSC & Customers & VRPSCD & Customers and Demands \\
\hline VRPSD & Demands & VRPSTT & Travel Times \\
\hline
\end{tabular}

VRP models usually have many applications; being solved by a great variety of heuristic techniques; among which we can mention stochastic, evolutionary, physical, swarm and neural algorithms, described and classified in greater detail in [16]. Table 2 shows the VRP models and the most used solution techniques. 
Table 2. Statistics of existing VRP models and the ir solution techniques; source: source [46].

\begin{tabular}{llllllll}
\hline Applied Method & $\begin{array}{l}\text { Number } \\
\text { Models }\end{array}$ & of & $\begin{array}{l}\text { Previous } \\
\text { Work }\end{array}$ & \multicolumn{2}{l}{ Number of Articles } & & \\
\cline { 4 - 7 } & & 2013 & 2014 & 2015 & 2016 & 2017 \\
\hline Classical Heuristics & 10 & 2 & 2 & 2 & 1 & 2 & 2 \\
Metaheuristic & 72 & 17 & 17 & 5 & 11 & 13 & 19 \\
Hybridation & 31 & 3 & 3 & 2 & 9 & 10 & 5 \\
Exact Method & 22 & 7 & 7 & 2 & 0 & 3 & 4 \\
Simulation & 9 & 4 & 4 & 1 & 0 & 3 & 1 \\
Adaptative Algorithms & 5 & 2 & 2 & 1 & 1 & 0 & 0 \\
& & & & & & &
\end{tabular}

Figure 1 shows the statistics of different articles analyzed for the elaboration of this investigation; they are classified by different applied methods.

This collection of works adds to the one previously made by [15] where a new classification of hybrid and adaptive algorithms that the authors include within the metaheuristics is attached.

Number of papers based on the solution method

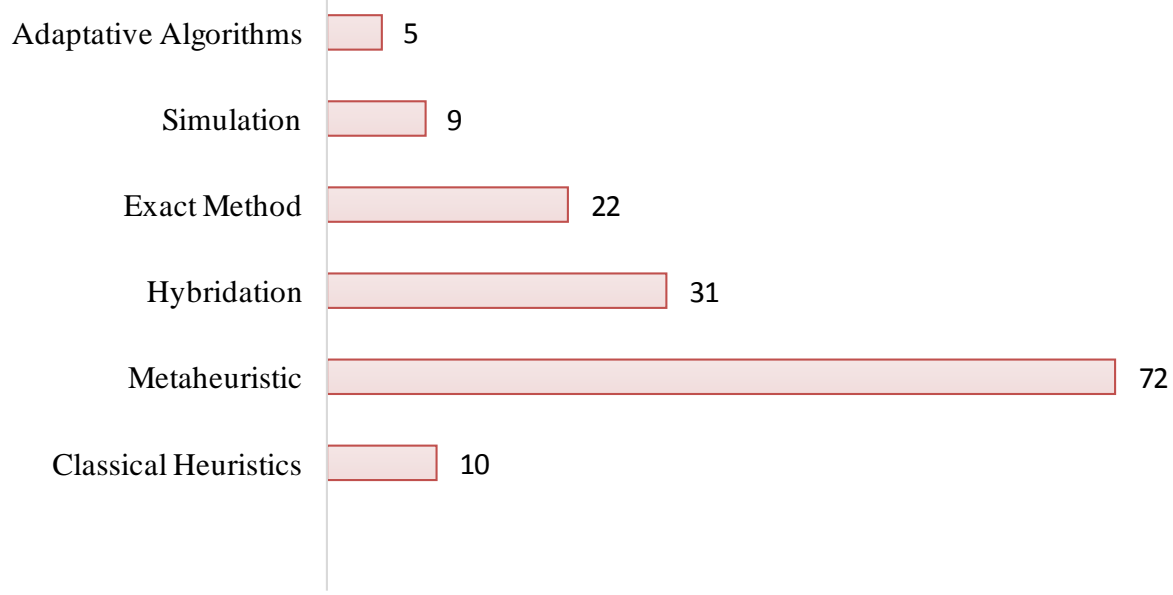

Figure 1. Preference of the use of the different techniques to solve VRP models, information of table 2.

Furthermore, it is important to mention that the process of hybridization is not only linked to the process of metaheuristics with metaheuristics; as mentioned in [11], it can also be presented between exact and metaheuristic methods. An important feature of these hybridization methods lies in improving the performance of the final algorithm, since it will combine the best individual characteristics of its predecessors, mitigating the opposite effects when hybridized $[13,56]$. These characteristics make this new technique one of the preferred ones to face the computational complexity that arises when incorporating a greater level of detail to the VRP. As seen in the work carried out by $[1,2,4,6,7]$.

In terms of simulation, there are different contributions depending on the characteristics of the model. for example, for stochastic models, there are simulations that use Monte Carlo simulation or Bayesian networks to explain the behavior of stochastic variables $[28,32]$. On the other hand, there are simulations based on agents that analyze the system and propose an $\mathrm{ABS}$ to solve dynamic vehicle routing, where the dynamic implies variation in the freight demand. Two types of agents are considered: retailers and truck drivers. The optimization problem is formulated in terms of traveled distance minimization. Retailer behavior is simulated with a lateness tolerance which is used as a constraint of the vehicle routing 
problem. The search algorithm for vehicle routes is the particle swarm [52]. Secondly [27] present the problem of location and routing of vehicles with transportation costs stochastic displacement speeds. The authors propose a hybrid solution procedure based on the optimization of ant colonies (ACO) and discrete event simulation (DES). Again, it can be seen here other type of hybrid models, optimization and simulation. After using a sequential heuristic algorithm to solve the location subproblem, the problem of routing the capacitated vehicle is solved using ACO.

During the last years, other techniques that have shown a remarkable growth in solving problems with a high level of difficulty, as shown in Table 3 and Figure 2, are the swarm algorithms. These metaheuristic algorithms have the characteristic of guiding a group of individuals through a region of solutions, where there is an individual and group search. Each individual shares search information with the rest of the swarm, which is moving towards that region where the solution tends to a global optimal $[3,8,20,40,42]$.

Table 3. Statistics based on the type of metaheuristics; source: [46].

\begin{tabular}{|c|c|c|c|c|c|c|c|}
\hline \multirow{3}{*}{ Kind of Metaheuristic } & \multirow{3}{*}{$\begin{array}{l}\text { Number } \\
\text { of Arti- } \\
\text { cles }\end{array}$} & \multicolumn{6}{|c|}{ Number of Articles } \\
\hline & & Previous & & & & & \\
\hline & & Work & 2013 & 2014 & 2015 & 2016 & 2017 \\
\hline Stochastic Algorithms & 34 & 7 & 1 & 4 & 4 & 6 & 12 \\
\hline Evolutionary Algorithms & 11 & 2 & 1 & 2 & 1 & 2 & 3 \\
\hline Physical Algorithms & 11 & 1 & 2 & 1 & 2 & 3 & 2 \\
\hline Swarm Algorithms & 16 & 7 & 1 & 0 & 4 & 2 & 2 \\
\hline
\end{tabular}

\section{Percentage of the number of papers classified by type of metaheuristics used}

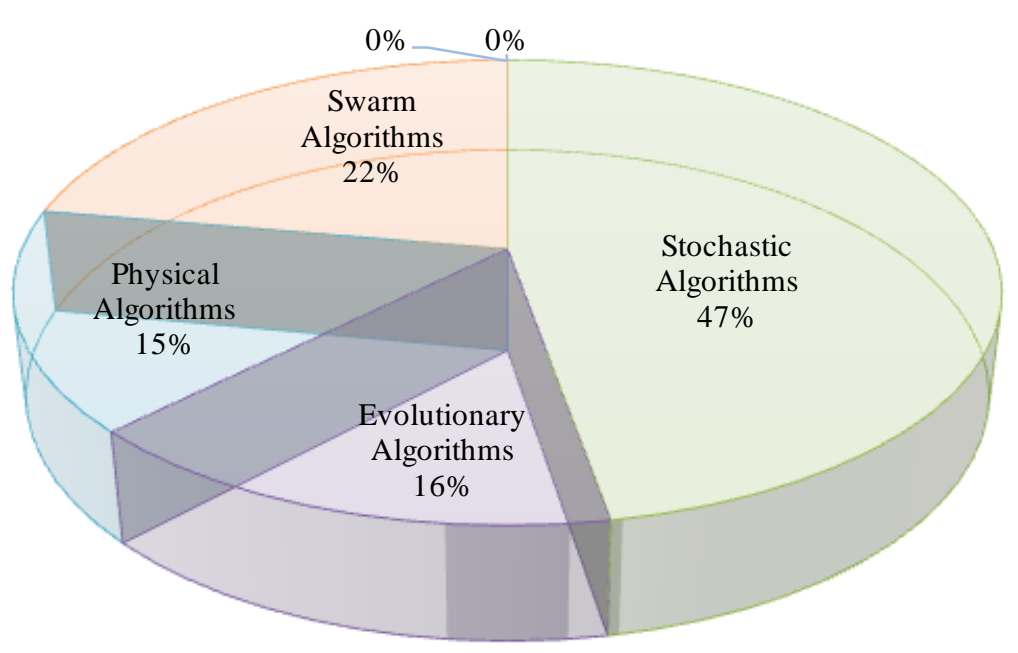

Figure 2. Preference of the use of the different metaheuristic algorithms.

\subsection{Complex networks and VRP}

A complex network is one whose structure has very peculiar characteristics that can be identified from certain very specific metrics; such as: number of node degree, levels of grouping, clustering coefficient and power law, among others [23]. In the literature, three types of complex networks are commonly mentioned, which are usually the most common to identify in real cases. 
In the first case, random networks are found. These networks have mainly two characteristics. The first of them refers to the distribution of (their nodes degree) the degrees of their nodes that can behave as a Binomial or Poisson distribution. In this case, the value of probability $p$ must be between 0 and 1 . The second characteristic is that the number of degrees of a node always oscillates near the average of degrees of the entire network, making it impossible to identify communities [50].

One method for the generation of random networks is the Erdös-Rényi model. In this type of model, there is the peculiarity that a node within the network will have the same probability of being connected to the other elements of the network. This probability is presented independently for each of the elements of the network. An example of this type of network can be identified in Figure 3.

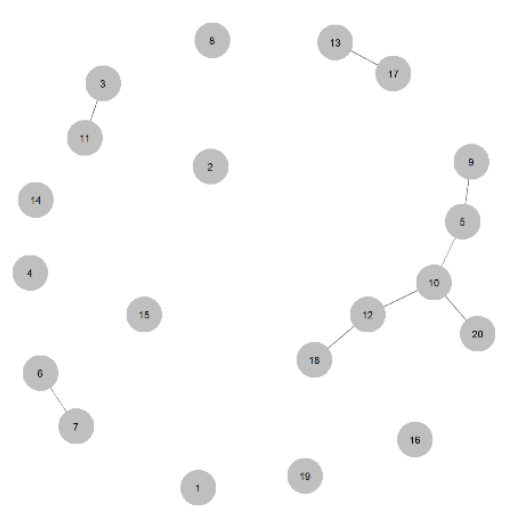

Figure 3. Example of a random network. Source by authors.

The second type of complex network is scale-free networks. The distribution of these networks shows that the degrees of their nodes behave like a power law with a probability $p(k)$ that a node of the network is connected with $k$ nodes and is proportional to $k^{-\alpha}$ that implies $p^{k} \sim k^{-\alpha}$, The exponent is not unique, but depends on the specific type of network. For most systems it was found that the parameter $\alpha$ is between $2 \leq \alpha \leq 3$ [23]. In this way it can be said that there are many nodes with a lower grade level, as shown in Figure 4.

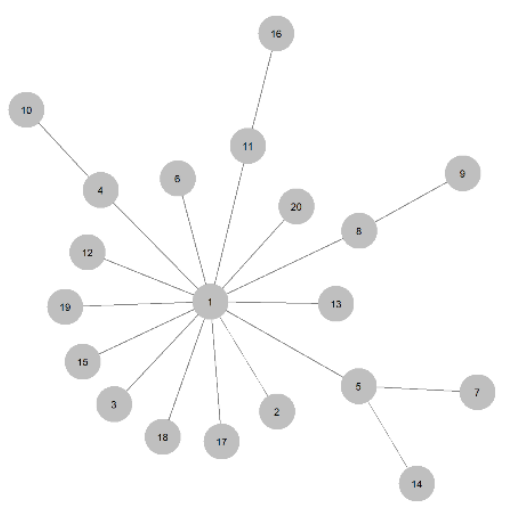

Figure 4. Example of Free-Scale networks. Source: by authors.

Finally, the small world networks have two characteristics that are:

- Any two nodes in the network communicate over a relatively small intermediate nodes path (small number of nodes). Noting that the maximum distance between two nodes grows logarithmically with the number of nodes in the network.

- They have high values of clustering coefficient (greater than one). This value indicates that if two nodes are not directly 
connected to each other there is a high probability that they are connected through the intervention of other nodes. And they have a shorter average route distance close to one [50].

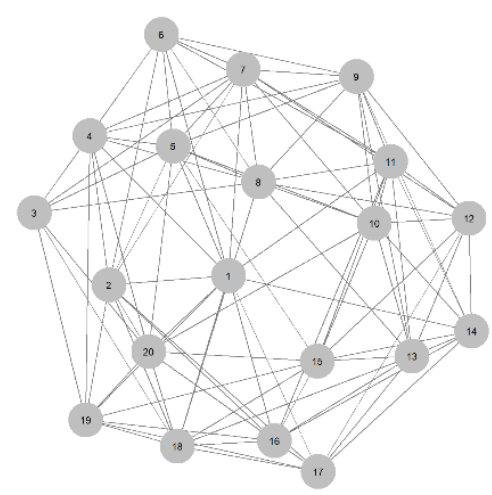

Figure 5. Example of Small-World networks. Source: by authors.

If it can be demonstrated that the distribution network of a company maintains a scale free or small world network behavior, then it can be analyzed as a network with a complex structure. So, it is convenient to answer the question: What are the advantages of doing a topological analysis of transport networks?

One of the main reasons to perform a topological analysis is the analysis of vulnerability and the detection of network clusters. The first study allows to identify the level of efficiency of the network against possible failure scenarios of any of its components. The second identifies groups with similar characteristics.

Consider the case of a company that has three different types of customers with different characteristics among them. A cluster analysis will identify how different the groups are and generate a routing plan for each of them, which is more like their characteristics, avoiding integrating them into a generic model that considers each one of the characteristics of the groups, but adding more computational complexity to solve the problem. In this way, it will be possible to divide the complexity according to the characteristics of the elements that make up the network.

Another way to visualize complex networks is the proposal of [12,35]; who make use of the concept of hierarchies and layers to represent schematically the operation of a complex system; allowing with this structure to visualize the different types of interrelationships at different levels of the system.

In [55] is mentioned that the logistic distribution system is a nonlinear dynamic system, which includes multilevel, multifunctional, multi-objective and multiple actors subject to many random factors. This feature, coupled with the fact that companies are increasingly immersed in a globalized market that requires constant logistic optimization to maintain competitive levels, as well as real-time distribution and routing, make imperative the use of devices such as GPS with routing. In the analysis that is proposed, the different relationships that exist between the agents of the network are considered. These relationships, as mentioned in [38], are attributes associated with the nodes and generate multiple types of relationships that are represented by arcs.

To understand the concept of complex network proposed by [12,35] mentioned above, first, it is necessary to start from the definition of a regular network. This can be defined as a network $G=(V, E)$, where $V=\left\{n_{1}, n_{2}, \cdots, n_{v}\right\}$ is the set of nodes and $E \subseteq V \times V=\left(e_{1}, e_{2}, \cdots, e_{k}\right)$ is the set of arcs connecting each pair of nodes $(i, j)$ where $i, j \in V$ y $i \neq j$ $[19,33,50]$.

From this definition it follows that the subscripts $v$ and $k$ are equivalent to the maximum number of nodes and arcs. On the other hand, the arc that joins two nodes $i$ and $j$ within the network, can be defined by $e_{i j}$; Some basic concepts of networks are mentioned by [30,49,50]. There are different definitions of a complex network; like the one by [12]; authors identify the layers of a complex network as a phase change of the product that flows through it. Proposal that has 
been used in urban transport, where a change in the mode of transport is a phase change and therefore a layer [5,25].

However, in this research proposal, due to the conditions of the VRP it cannot be analyzed as a multilayer structure. The concept of complex network is developed with the purpose of carrying out the analysis of the distribution network, according to each attribute in the model variables. In this way, it is possible to appreciate the indicators of the network, for each of these factors.

Below is the definition of VRP based on.[12,38,41]:

Definition: Let $G=(V, E)$, be a network where $V=\{0,1, \ldots, i\}$ is the set of nodes, where $\{1, \ldots, i\}$ represents the set of clients, and node 0 represents the store central. It can be more nodes as stores and they must be marked as such ones.

Each node $i \in V$ is associated with a set of attributes. $\alpha \in\{1, \ldots, A\}$; such as $i=\left(\right.$ Attribute $_{1}$, Attribute $_{2}, \ldots$, Attribute $\left._{\alpha}\right)$.

At least one of the attributes of the set of clients must represent its demand $d_{i}$; whereas the first attribute of the node must be the number that identifies the node within the network.

The set of $\operatorname{arcs} E$ can be defined as $\left(i_{\alpha}, j_{\beta}\right)$; where $j \in V$ and $i_{\alpha} \neq j_{\beta}$; for all $\alpha, \beta \in A$. In this case arc $E$ exists if and only if $\alpha \cap \beta \neq \emptyset$. Similarly, there may be a homogeneous or heterogeneous vehicle fleet in the model $m \in M$ with capacity $Q$ and a set of attributes (Attribute ${ }_{1}$, Attribute $e_{2}, \ldots$, Attribute $_{\gamma}$ ) to serve a set of clients.

In this way, the adjacency matrix is expressed as follows:

$$
a_{i j}^{\alpha, \beta}=\left\{\begin{array}{c}
1 \text { if }\left(i_{\alpha}, j_{\beta}\right) \in E \\
0, \text { de otra forma }
\end{array}\right.
$$

It is important to mention that in the adjacency matrix that a node $i$ to be linked to a node $j$ must share at least one attribute in common.

However, the arc generated $\left(i_{\alpha}, j_{\beta}\right)$ can exist in the system if and only if there is a cost $c_{i j}^{\alpha, \beta}$ that represents that connection through that common attribute. Duplication of attributes should be avoided, as is the case of costs. The attributes of the node can be expressed as capabilities in case of representing warehouses.

The metrics of complex networks that can be applied to the analysis of the distribution network can be found in [12]. Some of these metrics can be clusters, centralities, and node degrees, among others.

In [38] is mentioned that a distribution network is composed of many attributes that give personality to each of its elements, and that are important to understand their place and behavior within the network. As an example, in a social network there are multiple types of agents or individuals with individual and collective characteristics and that according to the attribute that you want to analyze, can generate a different interaction network [12,19,53].

Simulation has worked well in the conceptualization of complex systems [5, 25] in particular, agent-based simulation, as mentioned in [52]. This allows to identify the emergencies that arise from the interaction of the elements that make up the complex system [31]. The simulation techniques, as mentioned in [18] help to build and analyze different scenarios but to optimize it is necessary to use the simulation-optimization hybridization; that can be defined as the process to locate the best values for the input variables without an exhaustive exploration in a set of solutions. The same author identifies a set of optimization techniques that can be mixed with the simulation process. The latter will be used to obtain different scenarios of system states.

In the review of the state of art of VRP, no articles were identified that made use of complex networks to characterize or analyze the distribution network as shown in table 4 and figure 6. This lack of VRP analysis through complex networks can be understood as [21] mention: The objective of these models is the general optimization of resources to obtain a solution. This premise can be justified by the fact that most applications focus on reducing execution times and improving solutions through the application of metaheuristic methods that provide these two characteristics; as shown in the following table and figure. 
Table 4 Analysis of the use of complex and regular networks based on the state of the art, own design.

\begin{tabular}{llllllll}
\hline Transportation & Net- & Total Number of & Previous & & Number of Papers & & \\
\cline { 4 - 7 } work Design & Papers & Work & 2013 & 2014 & 2015 & 2016 & 2017 \\
\hline Regular Network & 184 & 89 & 13 & 21 & 22 & 17 & 17 \\
Complex Network & 0 & 0 & 0 & 0 & 0 & 0 & 0 \\
\hline
\end{tabular}

Total of Articles 184

Techniques used to solve the VRP

Other Techniques

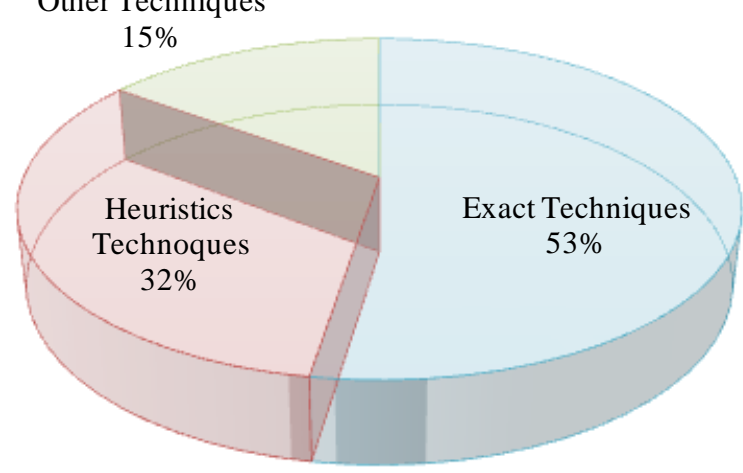

Figure 6. Techniques used to solve VRP.

In conclusion, the VRP models focus on reducing the computational complexity of the model; and complex networks approach is not considered. From the supply chain point of view routing problems are more complicated and demanding due to the fact that restrictions are added as companies grow and move towards a more globalized and competitive world $[34,54]$. This growth requires a general knowledge of the entire distribution network.

\section{Methodology}

Methodology shown in figure 7 is based on [9]. 


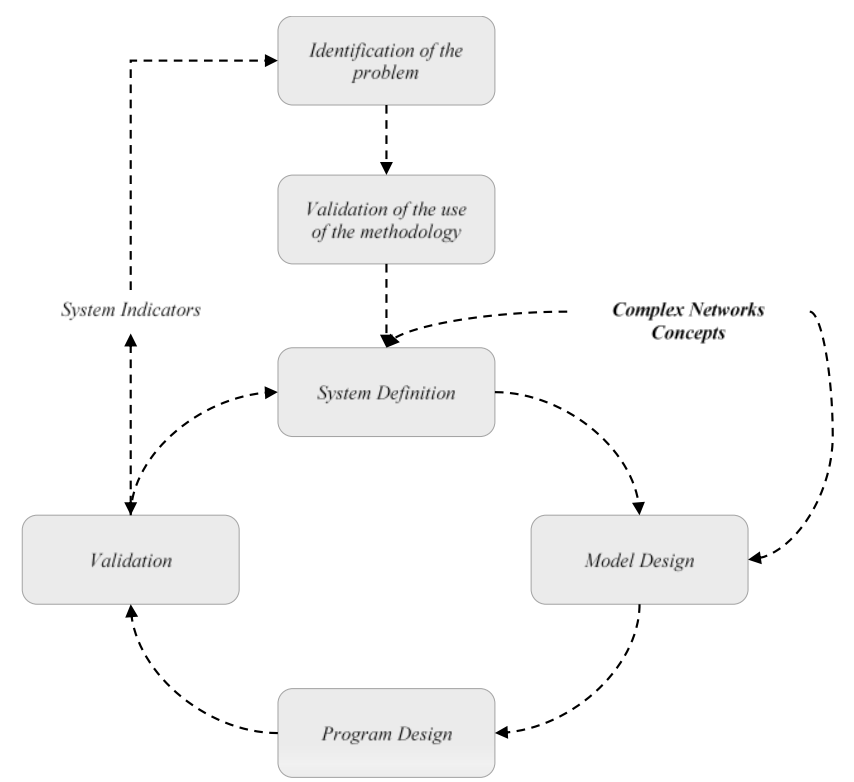

Figure 7. Generic scheme of the methodology. Adapted from [9].

The specific structure of the methodology is shown in Figure 8.

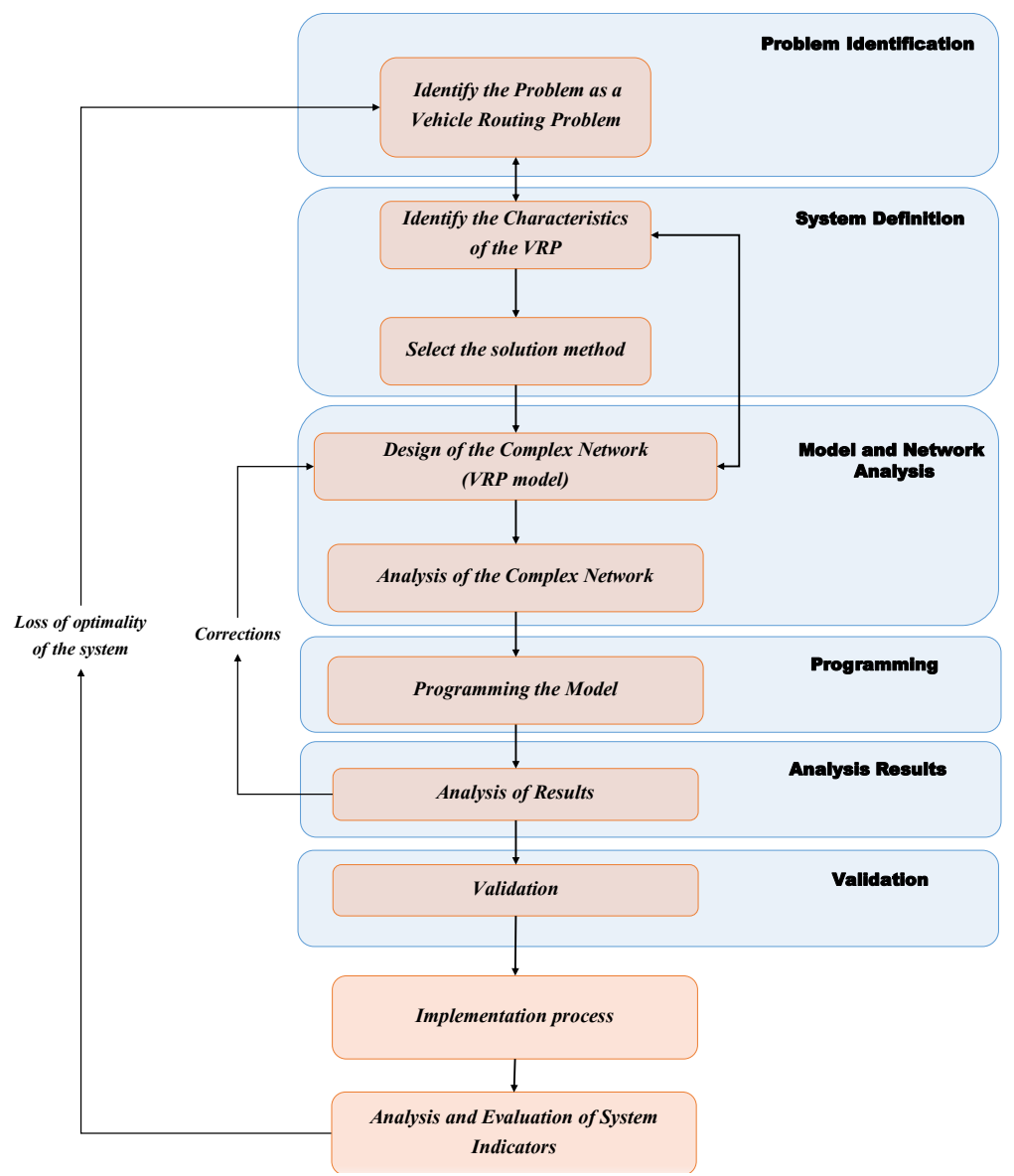

Figure 8. Proposed methodology. Source: by authors. 


\section{Step 1 Problem identification}

It is important in this step to identify the key logistic indicators related to the product distribution system. In order to detect potential breaches that may directly affect customer satisfaction or generate a cost to the company $[29,47]$. In the absence of such indicators, they must be defined.

Once a failure is detected through the indicators, it must be identified if the failure is attributable to the company's routing system, if so, continue with the next step. Otherwise stop and check the problem in other part of the system.

\section{Step II System definition}

In this second step, through a systemic analysis [47], all the elements and interrelations that directly participate in the product distribution process will be identified. Identifying the different types of nodes, arcs and flows within the network. See figure 9.

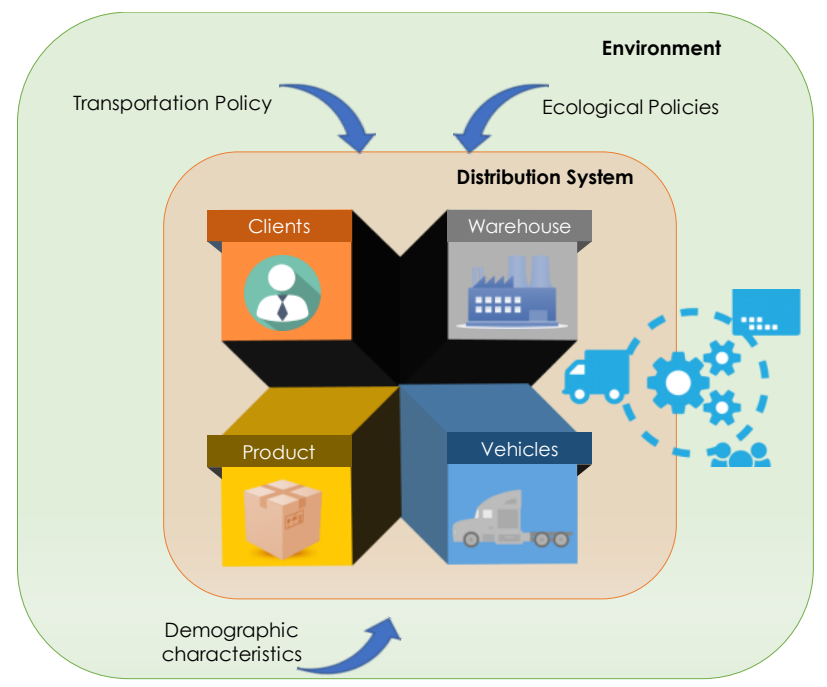

Figure 9. Systemic visualization of a supply chain. Source: by authors.

An important characteristic within this step, and that will differentiate the result with respect to other transport models existing in the literature, is the ability to add attributes to each of the elements of the network.

Consider the distribution system as a system with its respective environment in which, the role of the environment consists of requiring each of the elements (Customers, Stores, Vehicles and Products) of the network a set of restrictions that can be assigned as attributes to these same elements in a particular way; in such a way, that the elements close to each other, are expected to retain similar characteristics. In Figure 10, a brief example of the attributes associated with customers and vehicles is shown. 


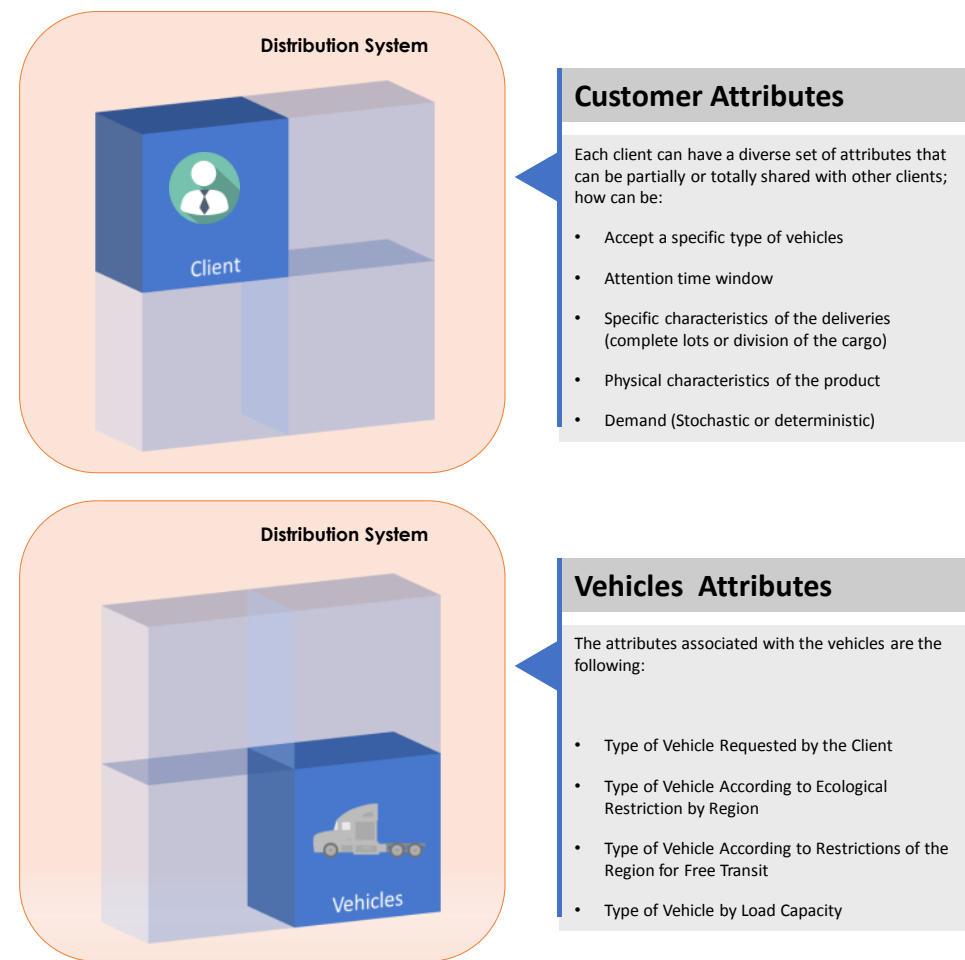

Figure 10. Brief example of attributes associated with customers and vehic les. Source: by authors

It is important to keep in mind that there may be two main types of attributes in the system; those that act as a restriction on the problem, and those that generate new objectives; As shown in figure 11.

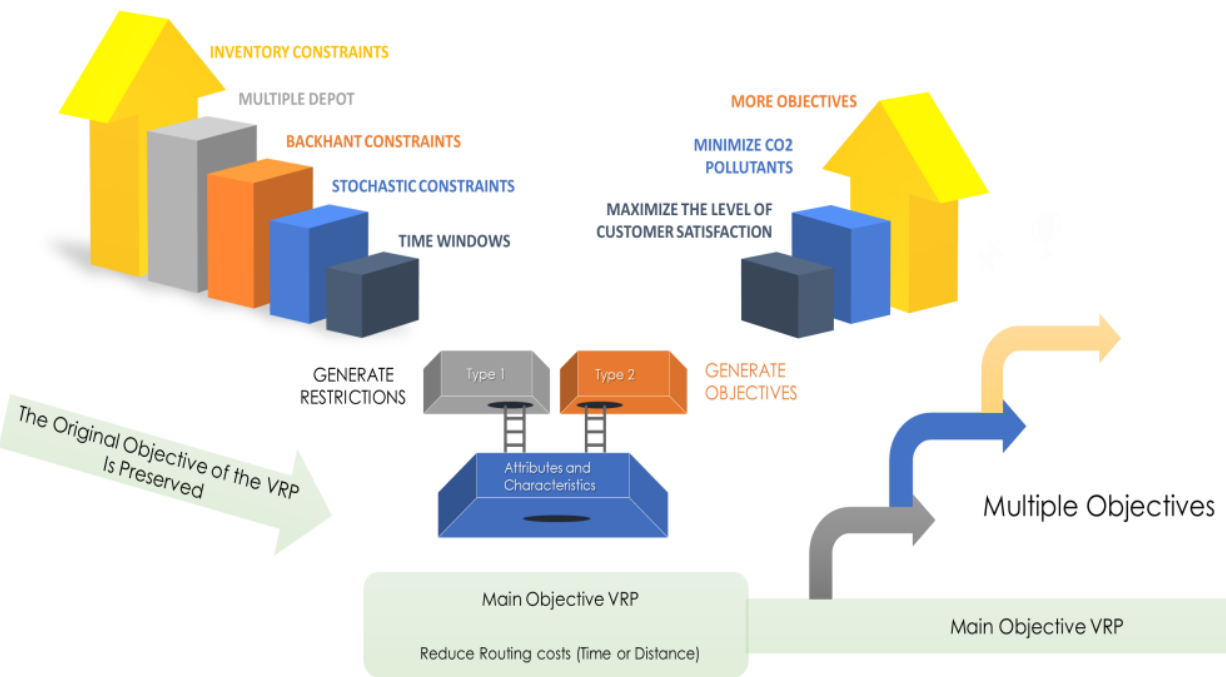

Figure 11. Classification of different types of attributes. Source: by authors.

Figure 12 shows some characteristics or attributes that can be associated to the different elements of the network in terms of transport models. These characteristics will increase restrictions under the same objective, which is the minimization of the route. But there may be attributes that match the objective function of the VRP in terms of minimization, such as minimizing the level of contamination by $\mathrm{CO}_{2}$, in which case it can be modeled as a multi-objective if necessary. 


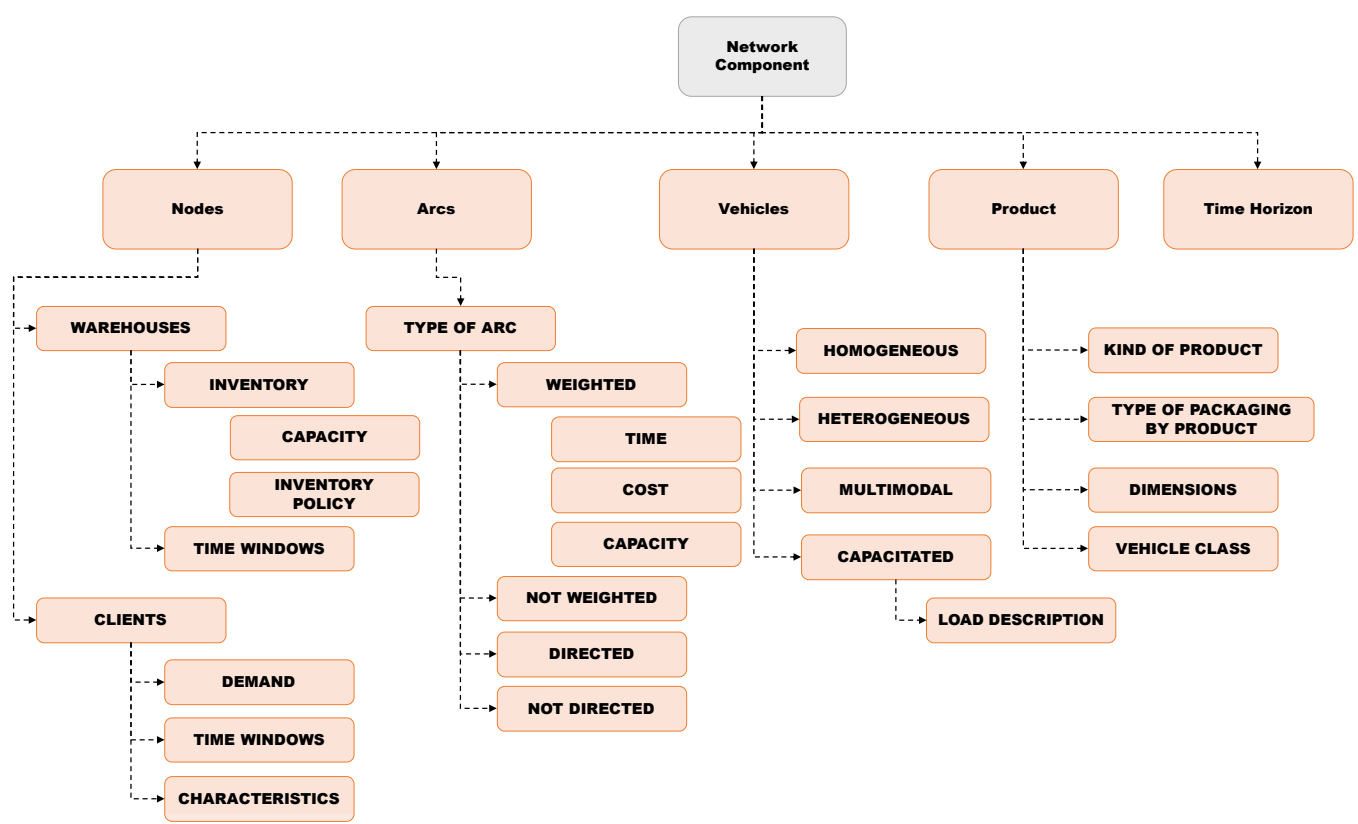

Figure 12. Elements of a VRP network, Source: by authors.

With this information, it is possible to characterize the corresponding VRP model; using the classification shown in figure 13 .

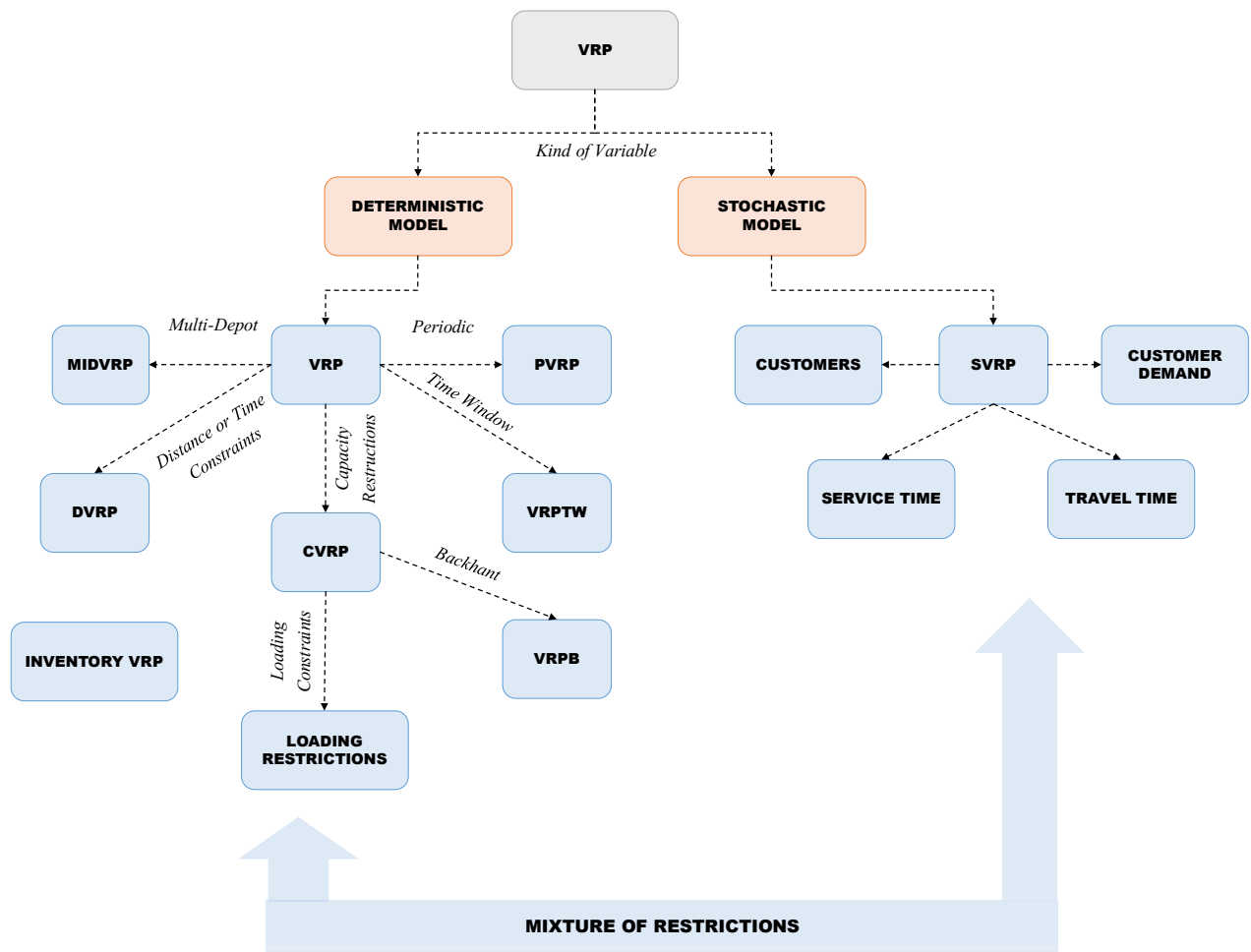

Figure 13. VRP classification; based on: [10,41]. 
It is necessary to consider that from the attributes of the nodes or elements of the network it is possible to generate subgroups with common characteristics. These groups can generate different models of VRP with which the problem can be divided into subproblems of smaller networks and therefore more manageable in terms of their data structure as shown in Figure 14.

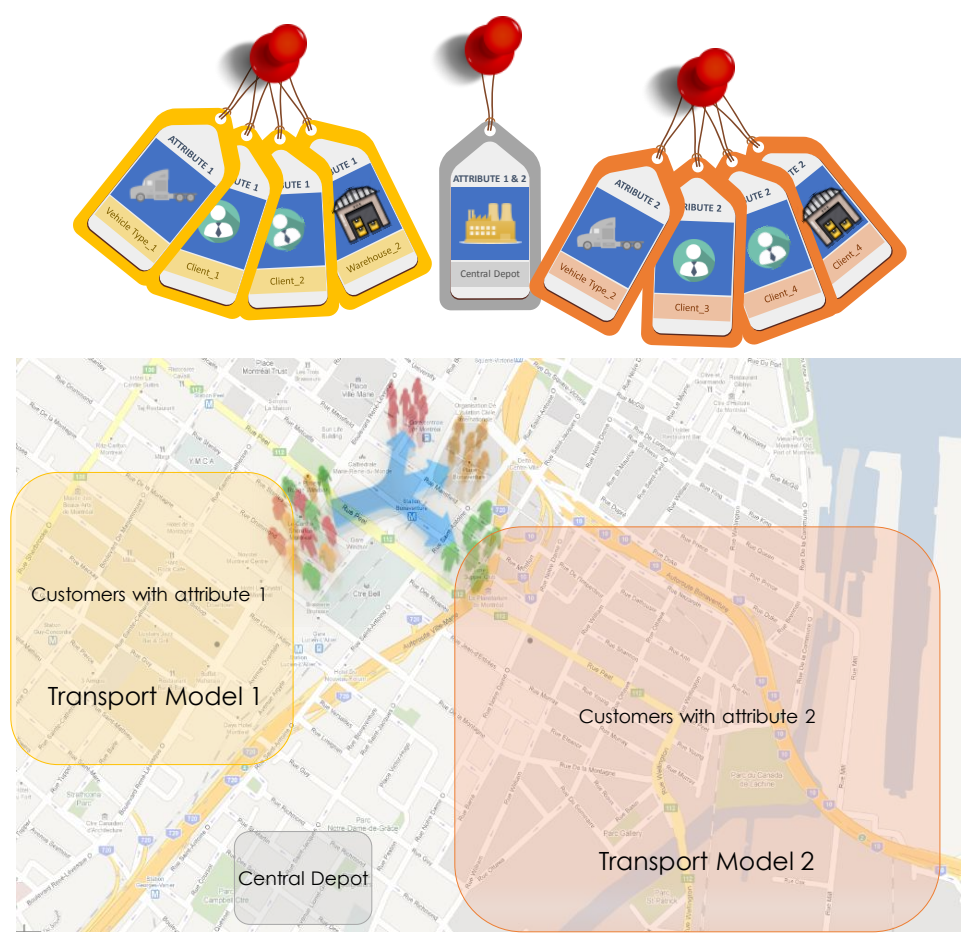

Figure 14. Identification of the VRP subgroups based on the attributes. Source by authors.

Once the general VRP and its subsets have been characterized, it is possible to identify suitable algorithms to optimize and simulate to construct different scenarios.

\section{Step III Design and analysis of the complex network (VRP Model)}

The creation of the distribution network is generated by identifying the nodes and the respective arcs of union between them. It is important to mention that, due to the existence of different attributes, a node can be duplicated if it shares characteristics or variables with another group to which it belongs. In this way, the joining arc between a duplicated node and the other nodes must be black; and in turn, it must include a phase change function between one attribute and another. This concept is used by multilayer transport models, shown by [5,25]. The authors duplicate the nodes when there is a change of transport mode and joining these duplicate nodes through a transfer arc that transforms the variable from one specific attribute to another. For example, if a person is in a public transport network such as the subway, and changes to a taxi, the time that elapses in the change of transport is the transfer arc, in which the attributes of that person shifts to the taxi transport network. In this same way, there may be attributes that share variables; and now that a variable is auxiliary in the calculations of another attribute, it changes phases starting from a function. See figure 15. 


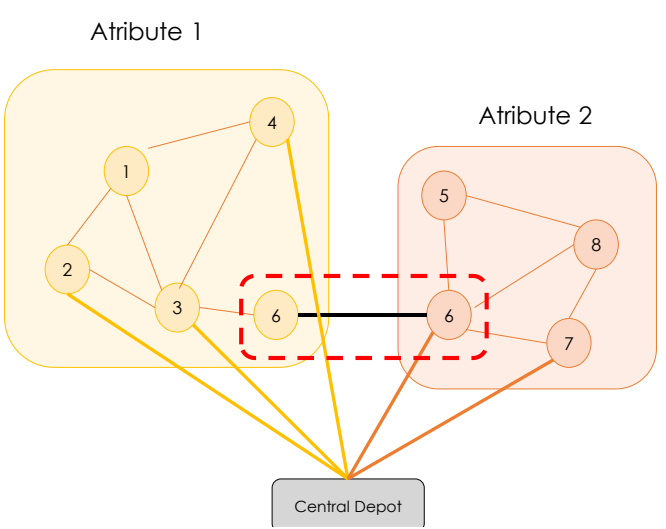

Figure 15. Distribution network based on [12].

The analysis of the distribution network will allow to identify the level of vulnerability of the network to possible failures and alternate routes among others. In the same way, it will validate the subgroups created from different attributes. With the vulnerability analysis of the network, the different simulation scenarios can be executed.

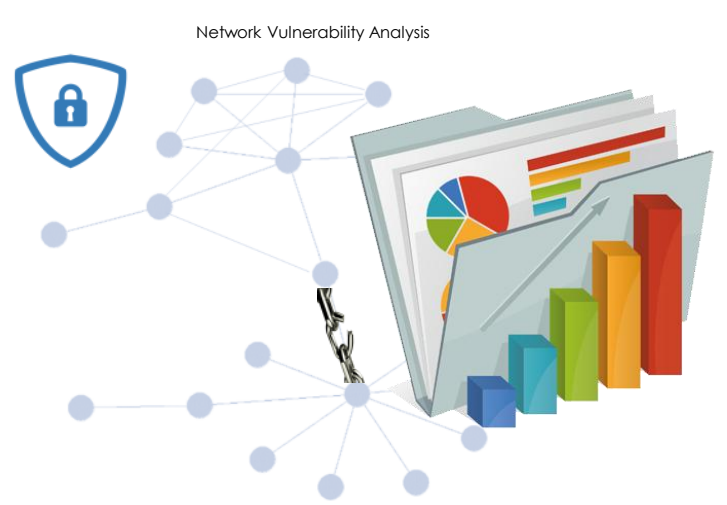

Figure 16. Analys is of the distribution network. Source: by authors.

\section{Step IV Model programming}

In this step, the model is programmed in an agent-based simulation software because of the compatibility with complex networks, they can be Anylogic or SIMIO.

\section{Step V Simulation and results analysis}

In this step, the simulation and definition of the different scenarios are executed, looking for the vulnerability of the network and its solution.

\section{Step VI Validation and Verification}

In this step, the results must be validated by comparing the results with respect to real data or with small examples of other similar models; as mentioned in [31].

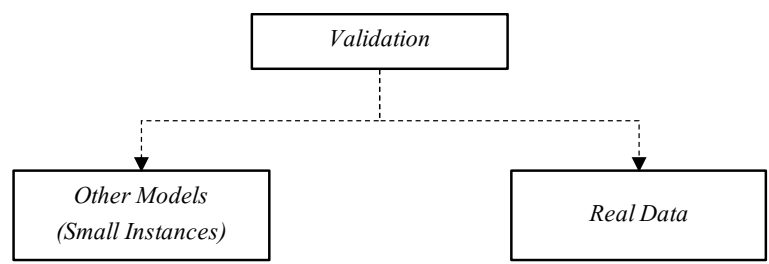

Figure 17. Validation modes. Source: by authors. 
Step VII Analysis and evaluation of indicators

The indicators corresponding to the distribution system identified in Step I must be constantly monitored to verify their correct operation. In the case of detecting any defect or improvement in the system, the process is restarted from Step I.

\section{Step VIII Operational plan}

At this step is important to build an action plan, technical reports and guides for the users who will oversee the system.

\section{Expected Results and Future Work}

With the application of the methodology, it is expected to be able to design more robust VRP models that consider the variability of the real systems and thus detect possible problems of supply and failures to be able to propose the optimization and construction of scenarios through simulation.

The research is at the level of the methodology that shows advantages, scope and limitations and makes possible corrections to any of its steps. Future work contemplates the application to a real case.

\section{Conclusion}

Based on the analysis carried out, there is a great concern about the limitation of resources in the design of VRP models. These limitations are usually the execution times, or the quality of the solutions expected for the decision maker. However, it should not be forgotten that the main objective of a VRP is to solve a real-life problem, where multiple variables converge with multiple characteristics and attributes that can be significant and whose omission results in a model that is not real [14].

For this reason, modelers are encouraged to expand the details of their models to obtain a broader knowledge of what happens within the distribution network.

\section{Acknowledgments}

We are grateful for the support to CONACYT for making this work possible; likewise, we greatly thank to our tutor Dr. Idalia Flores de la Mota whom we greatly esteem.

Special thanks to the PAPIIT project IT102117 "Accesibilidad y movilidad del transporte público urbano en la Ciudad de México, caso de la delegación de Tlalpan".

\section{Authors Biography}

Gabriel Policroniades Chípuli: Studied Industrial Engineering at Technological Institute and Superior Studies of Monterrey, Campus Toluca (ITESM, Campus Toluca), he studied an engineering mastery with operation research orientation and has advanced metrology studies for the calculation of uncertainty, and knowledge of Lean Six Sigma Black Belt and Logistics and supply chain Currently he is studying a Ph.D. in Operation Research at UNAM.

Idalia Flores de la Mota: She received her Ph.D. in Operations Research at the Faculty of Engineering of the UNAM. She graduated Master with honors and received the Gabino Barreda Medal for the best average of her generation. She has been a referee and a member of various Academic Committees at CONACYT. She has been a referee for journals such as Journal of Applied Research and Technology, the Center of Applied Sciences and Technological Development, UNAM and the Transactions of the Society for Modeling and Simulation International. Her research interests are in simulation and optimization of production and service systems. She is a full-time professor at the Postgraduate Program at UNAM.

Sashiko Shirai: obtained her bachelor's in actuarial science in the Faculty of Science of the UNAM. She is studying her Master in Operations Research in the Faculty of Engineering of the UNAM with the CONACYT scholarship. She is an assistant professor at the Faculty of Engineering and the Faculty of Science. Her research interests lie in complex networks, statistics and operational research. 
Javier Lara de Paz: studied Physics at the Faculty of Science of National Autonomous University of Mexico (UNAM), a Specialization in Applied Statistics at the UNAM. He studied his master's degree in Operation Research at the Faculty of Engineering of the UNAM. He has been professor of Science at the and Operation Research professor at the Engineering School at the UNAM. Currently he is studying a Ph.D. in Operational Research at UNAM.

\section{References}

[1] M.M.S. Abdulkader, Y. Gajpal, T.Y. ElMekkawy, Hybridized ant colony algorithm for the Multi Compartment Vehicle Routing Problem, Applied Soft Computing. 37 (2015) 196-203.

[2] A. Agra, C. Requejo, F. Rodrigues, A hybrid heuristic for a stochastic production-inventory-routing problem, Electronic Notes in Discrete Mathematics. 64 (2018) 345-354.

[3] T.J. Ai, V. Kachit vichyanukul, A particle swarm optimization for the vehicle routing problem with simultaneous pickup and delivery, Computers \& Operations Research. 36 (2009) 1693-1702.

[4] S. Akpinar, Hybrid large neighbourhood search algorithm for capacitated vehicle routing problem, Expert Systems with Applications. 61 (2016) 28-38.

[5] A. Aleta, S. Meloni, Y. Moreno, A multilayer perspective for the analysis of urban transportation systems, ArXiv: 1607.00072 [Cond-Mat, Physics:Physics]. (2016).

[6] A. Alvarez, P. Munari, An exact hybrid method for the vehicle routing problem with time windows and multiple deliverymen, Computers \& Operations Research. 83 (2017) 1-12.

[7] M. Avci, S. Topaloglu, An adaptive local search algorithm for vehicle routing problem with simultaneous and mixed pickups and deliveries, Computers \& Industrial Engineering. 83 (2015) 15-29.

[8] D. Baeza, C.F. Ihle, J.M. Ortiz, A comparison between ACO and Dijkstra algorithms for optimal ore concentrate pipeline routing, Journal of Cleaner Production. 144 (2017) 149-160.

[9] J. Banks, Wiley: Handbook of Simulation: Principles, Methodology, Advances, Applications, and Practice - Jerry Banks, Wiley, 1998.

[10]E. Berhan, B. Beshah, D. Kitaw, A. Abraham, Stochastic Vehicle Routing Problem: A Literature Survey, J. Info. Know. Mgmt. 13 (2014) 1450022.

[11]C. Blum, A. Roli, Hybrid Metaheuristics: An Introduction, in: Hybrid Metaheuristics, Springer, Berlin, Heidelberg, 2008: pp. $1-30$.

[12]S. Boccaletti, G. Bianconi, R. Criado, C.I. del Genio, J. Gómez-Gardeñes, M. Romance, I. Sendiña-Nadal, Z. Wang, M. Zanin, The structure and dynamics of multilayer networks, Physics Reports. 544 (2014) 1-122.

[13]A. Bortfeldt, A hybrid algorithm for the capacitated vehicle routing problem with three-dimensional loading constraints, Computers \& Operations Research. 39 (2012) 2248-2257.

[14]N. Boysen, S. Emde, M. Hoeck, M. Kauderer, Part logistics in the automotive industry: Decision problems, literature review and research agenda, European Journal of Operational Research. 242 (2015) 107-120.

[15]K. Braekers, K. Ramaekers, I. Van Nieuwenhuyse, The vehicle routing problem: State of the art classification and review, Computers \& Industrial Engineering. 99 (2016) 300-313.

[16]J. Brownlee, Clever Algorithms: Nature-Inspired Programming Recipes, Lulu.com, s.l., 2012.

[17]M. de Bucourt, R. Busse, F. Güttler, C. Wintzer, F. Collettini, C. Kloeters, B. Hamm, U.K. Teichgräber, Lean manufacturing and Toyota Production System terminology applied to the procurement of vascular stents in interventional radiology, Insights Imaging. 2 (2011) 415-423.

[18]Y. Carson, A. Maria, Simulation Optimization: Methods And Applications, in: WSC, 1997: pp. 118-126.

[19]D.J.P. Casasnovas, Some Basic Concepts on Complex Networks and Games, in: Evolutionary Games in Complex Topologies, Springer Berlin Heidelberg, 2012: pp. 9-46.

[20]M.-C. Chen, Y.-H. Hsiao, R. Himadeep Reddy, M.K. Tiwari, The Self-Learning Particle Swarm Optimization approach for routing pickup and delivery of multiple products with material handling in multiple cross-docks, Transportation Research Part E: Logistics and Transportation Review. 91 (2016) 208-226.

[21]J.-F. Cordeau, G. Laporte, M.W.P. Savelsbergh, D. Vigo, Chapter 6 Vehicle Routing, in: C.B. and G. Laporte (Ed.), Handbooks in Operations Research and Management Science, Elsevier, 2007: pp. 367-428.

[22] G.B. Dantzig, J.H. Ramser, The Truck Dispatching Problem, Manage. Sci. 6 (1959) 80-91.

[23]E. Estrada, Introduction to Complex Networks: Structure and Dynamics, in: J. Banasiak, M. Mokhtar-Kharroubi (Eds.), Evolutionary Equations with Applications in Natural Sciences, Springer International Publishing, 2015: pp. 93-131. 
[24]J. Faulin, A. Juan, F. Lera, S. Grasman, Solving the Capacitated Vehicle Routing Problem with Environmental Criteria Based on Real Estimations in Road Transportation: A Case Study, Procedia - Social and Behavioral Sciences. 20 (2011) 323-334.

[25]R. Gallotti, M. Barthelemy, The multilayer temporal network of public transport in Great Britain, Scientific Data. 2 (2015) sdata201456.

[26]A. Hämmerle, M. Ankerl, Solving a Vehicle Routing Problem with Ant Colony Optimisation and Stochastic Ranking, in: Computer Aided Systems Theory - EUROCAST 2013, Springer, Berlin, Heidelberg, 2013: pp. 259-266.

[27] N. Herazo-Padilla, J.R. Montoya-Torres, S.N. Isaza, J. Alvarado-Valencia, Simulation-optimization approach for the stochastic location-routing problem, J Simulation. 9 (2015) 296-311.

[28]F. Hernandez, D. Feillet, R. Giroudeau, O. Naud, Branch-and-price algorithms for the solution of the multi-trip vehicle routing problem with time windows, European Journal of Operational Research. 249 (2016) 551-559.

[29] Hernandez, R, METODOLOGIA DE LA INVESTIGACION, MCGRAW-HILL INTERAMERICANA, 2010.

[30]M.M. Hossain, S. Alam, A complex network approach towards modeling and analysis of the Australian Airport Network, Journal of Air Transport Management. 60 (2017) 1-9.

[31] A. Huerta Barrientos, Metodología basada en modelos de simulación para el análisis de sistemas complejos (MoSASCoM), Facultad de Ingenieria, UNAM, Ciudad de México, 2014.

[32] A.A. Juan, J. Faulin, E. Pérez-Bernabeu, O. Domínguez, Simulation-Optimization Methods in Vehicle Routing Problems: A Literature Review and an Example, in: Modeling and Simulation in Engineering, Economics, and Management, Springer, Berlin, Heidelberg, 2013: pp. 115-124.

[33] Katharina A. Zweig, Network Analysis Literacy - A Practical Approach to the Analysis of Networks, 1st ed., Springer-Verlag Wien, 2016.

[34] Khojasteh, Yacob, Supply Chain Risk Management - Advanced Tools, Models, and | Yacob Khojasteh | Springer, Springer, 2018.

[35]M. Kivelä, A. Arenas, M. Barthelemy, J.P. Gleeson, Y. Moreno, M.A. Porter, Multilayer networks, J Complex Netw. 2 (2014) 203-271.

[36] V.S. Kumar, M.R. Thansekhar, R. Saravanan, S.M. Joe Amali, Solving Multi-objective Vehicle Routing Problem with Time Windows by FAGA, Procedia Engineering. 97 (2014) 2176-2185.

[37]W.L. Lee, Real-Life Vehicle Routing with Non-Standard Constraints, Proceedings of the World Congress on Engineering \& Computer Science. (2013) 432-437.

[38]W. Lei, G. Mingfang, W. Lijun, The Directed Complex Network Application in the Supply Chain, in: 2012 Third International Conference on Digital Manufacturing Automation, 2012: pp. 911-914.

[39]J.K. Lenstra, A.H.G.R. Kan, Complexity of vehicle routing and scheduling problems, Networks. 11 (1981) $221-227$.

[40]M. Marinaki, Y. Marinakis, A Glowworm Swarm Optimization algorithm for the Vehicle Routing Problem with Stochastic Demands, Expert Systems with Applications. 46 (2016) 145-163.

[41] M.A. Mohammed, M.K.A. Ghani, R.I. Hamed, S.A. Mostafa, M.S. Ahmad, D.A. Ibrahim, Solving Vehicle Routing Pro blem by Using Improved Genetic Algorithm for Optimal Solution, Journal of Computational Science. (2017).

[42]N. Norouzi, M. Sadegh-Amalnick, M. Alinaghiyan, Evaluating of the particle swarm optimization in a periodic vehicle routing problem, Measurement. 62 (2015) 162-169.

[43]J. Oyola, H. Arntzen, D.L. Woodruff, The stochastic vehicle routing problem, a literature review, Part II: solution methods, EURO J Transp Logist. (2016) 1-40.

[44]S.N. Parragh, K.F. Doerner, R.F. Hartl, A survey on pickup and delivery problems, Journal Für Betriebswirtschaft. 58 (2008) $21-51$.

[45]S.N. Parragh, K.F. Doerner, R.F. Hartl, A survey on pickup and delivery problems, Journal Für Betriebswirtschaft. 58 (2008) 81-117.

[46] G. Policroniades, I.F. de la Mota, State of the Art of the Different Models of Transportation Most Used in the Supply Chain of Automotive Industry, 1.7 (2016) 44-53.

[47]Prawda, J., Metodos y modelos de investigacion de operaciones . Vol I-II, Decima, Limusa, 1991.

[48]Raghavan, S. Golden, Bruce L, The Vehicle Routing Problem: Latest Advances and New Challenges, 1st ed., Springer, 2008.

[49]S. Saha Ray, Graph Theory with Algorithms and its Applications, Springer India, India, 2013.

[50]T.C. Silva, L. Zhao, Complex Networks, (2016) 15-70.

[51]D. Solomon, M. J., Survey Paper-Time Window Constrained Routing and Scheduling Problems, Transportation Science. 22 (1988) 1-13. 
[52]B.M. Sopha, A. Siagian, A.M.S. Asih, Simulating Dynamic Vehicle Routing Problem using Agent-Based Modeling and Simulation, in: 2016 IEEE International Conference on Industrial Engineering and Engineering Management (IEEM), 2016: pp. $1335-1339$.

[53]M.T. Thai, P.M. Pardalos, eds., Handbook of Optimization in Complex Networks, Springer New York, New York, NY, 2012.

[54]X. Yang, A Review of Distribution Related Problems in Logistics and Supply Chain Research, International Journal of Supply Chain Management. 2 (2013) 1-8.

[55]T. Zhang, W.A. Chaovalitwongse, Y. Zhang, Integrated Ant Colony and Tabu Search approach for time dependent vehicle routing problems with simultaneous pickup and delivery, J Comb Optim. 28 (2014) 288-309.

[56]X. Zhang, L. Tang, A new hybrid ant colony optimization algorithm for the vehicle routing problem, Pattern Recognition Letters. 30 (2009) 848-855. 